\begin{abstract}
Wenn es um die Diabetesbehandlung der Zukunft geht, dürfen auch einmal Visionen artikuliert werden: Gelänge es, die Funktion der Betazellen des Pankreas auf Dauer zu erhalten, hätte sich das Problem des Typ-2-Diabetes erledigt, so der Pharmakologe Prof. Hans-Georg Joost aus Nuthetal. Aber was kann die Therapie von heute?
\end{abstract}

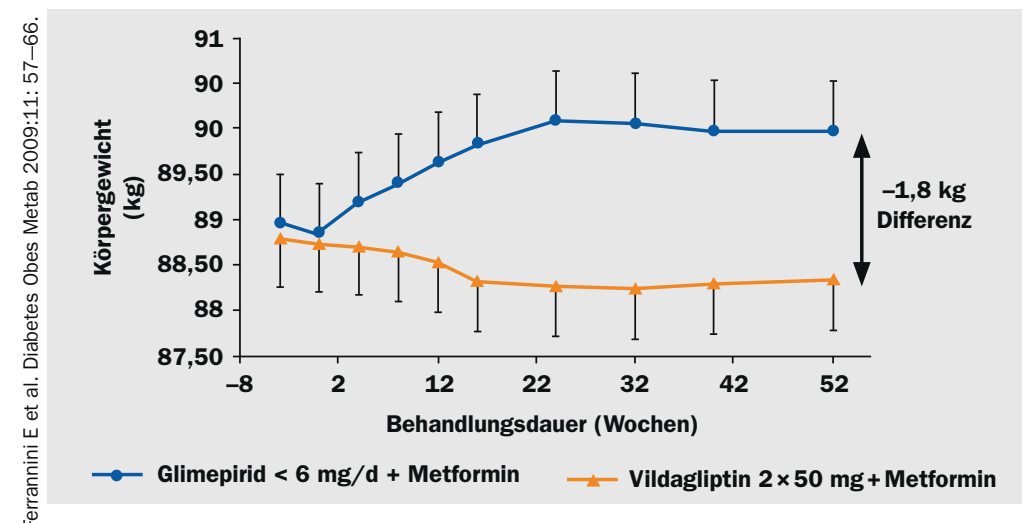

Abb. 1: Während die Patienten mit dem Sulfonylharnstoff zunahmen, nahmen jene mit der Gliptintherapie sogar leicht ab.

Beim Thema Inselzellschutz machen jene Medikamente Hoffnung, die über eine Anhebung der GLP-1-Konzentration wirken. So kann die für den Diabetes Typ 2 charakteristische Inselzellenresistenz gegenüber körpereigenen Inkretinen vermindert werden, so Prof. Wolfgang Schmidt, Bochum. Anheben lasse sich das GLP-1 entweder durch Hemmung des abbauenden Enzyms Dipeptidylpeptidase 4 (DPP 4) oder mit Inkretinmimetika.

Was diese Therapieansätze so attraktiv macht, ist nicht nur die moderate $\mathrm{HbA}_{1 \mathrm{c}}$-Senkung, sondern z.B. auch, dass man ihnen eine besondere Schonung der Betazellen zuschreibt. Die Hoffnung auf betazellprotektive Effeke stützt sich bislang vor allem auf Ergebnisse von Tiermodellstudien. Ob sie sich im Menschen zeigen, ist derzeit noch unklar.

Was man heute schon weiß: Inkretinbasierte Medikamente bergen im Gegensatz zu anderen insulinotropen Antidiabetika kein Hypoglykämierisiko. Unterzuckerungen sind laut Dr. Gerhard

\section{Kurz notiert}

\section{Insulinpumpenvertrieb geändert}

Die Animas Corporation, Hersteller der Insulinpumpen IR 1200 und 2020, hat den Vertrieb von Produkten in Deutschland geändert. Seit 1.1.2010 werden Animas Pumpen und Zubehör (Reservoirs, InfusionsSets etc.) exklusiv durch die Firmen DiaExpert und MediqDirekt vertrieben, bekannte nationale Anbieter von Diabetikerbedarf. Kunden, die in der Vergangenheit Pumpenzubehör von der Firma MedTrust bestellt haben, werden gebeten, sich für zukünftige Bestellungen an DiaExpert (www.diaexpert.de, Tel. 0800 3423973) und MediqDirekt (www.mediqdirekt.de, Tel. 0800 0560580) zu wenden.
Klausmann, niedergelassener Diabetologe in Aschaffenburg, ein entscheidendes Problem in der Diabetologie. Nicht zuletzt seien sie der Hauptgrund dafür, dass Therapieziele wie die Gewichtsabnahme oft nicht erreicht werden. Gegen Hypoglykämien äßen viele Patienten nämlich schlichtweg an. Bei DPP-4-Hemmern handelt es sich dagegen, anders als bei Sulfonylharnstoffen oder Insulin, um eine gewichtsneutrale Therapie, wie Dr. Bernd Donaubauer, Oschatz, mit Kasuistiken darlegte und wie auch Studien belegen (s. Abb. 1).

Dass mit Hypoglykämien unter DPP-4-Hemmern wie Vildagliptin (Galvus ${ }^{\circledR}$ ) nicht zu rechnen ist, hat folgenden Grund: Die Insulinfreistzung wird nur dann stimuliert, wenn die Blutzuckerspiegel erhöht sind. Fällt Glukose auf kritische Werte, steigen bei Gliptintherapie die Glukagonspiegel, eine Art „eingebauter Hypoglykämieschutz“ so Prof. Petra-Maria Schumm-Dräger, München.

Das Prinzip einer glukoseabhängigen Wirkung auf Alpha- und Betazellen ist gerade dann interessant, wenn man auch die postprandialen Blutzuckerspiegel in den therapeutischen Fokus nimmt. Dafür gibt es nach Einschätzung von Dr. Eva-Maria Fach gute Gründe. Die Diabetologin aus Rosenheim erinnerte damit an die Verlautbarungen der International Diabetes Federation (IDF).

Nach Meinung von Prof. Stefan Matthaei, Quakenbrück, könne man die postprandialen Blutglukose so lange hintenanstellen, wie der $\mathrm{HbA}_{1 \mathrm{c}}$ in Ordnung sei. „Liegt der $\mathrm{HbA}_{1 \mathrm{c}}$ im Zielbereich, hat der Patient keine Hypoglykämien, und gibt es keine Gewichtsprobleme, dann haben Sie gewonnen“ - so der Leitliniensprecher der Deutschen Diabetesgesellschaft (DDG). Den Einsatz von DPP-4Hemmern sehen die DDG-Leitlinien inzwischen bereits dann vor, wenn sich eine Monotherapie mit Metformin als unzureichend erweist.

Begrüßt wurde von den Experten, dass die aktuellen DDG-Leitlinien den Spielraum für eine individuelle Diabetestherapie lassen. Einer willkürlichen und restriktiven Festlegung auf Insulin, Sulfonylharnstoffe und Metformin als Allheilmittel in der Diabetologie erteilte PD Dr. Rainer Lundershausen, Erfurt, eine klare Absage.

urm

Expertenworkshop „Diabetestherapie der Zukunft“, 20. und 21.10.2009 in Montreal, Veranstalter: Novartis 